\title{
Numerical study of the pressure wave-induced shedding mechanism in the cavitating flow around an axisymmetric projectile via a compressible multiphase solver
}

\author{
Bingsheng Ye ${ }^{a, b}$, Yiwei Wang ${ }^{a, b, *}$, Chenguang Huang ${ }^{a, b}$, Jian Huang ${ }^{b}$ \\ ${ }^{a}$ Key Laboratory for Mechanics in Fluid Solid Coupling Systems, Institute of Mechanics, Chinese Academy of Sciences, Beijing, 100190, China \\ b School of Engineering Science, University of Chinese Academy of Sciences, Beijing, 100049, China
}

\section{A R T I C L E I N F O}

\section{Keywords:}

Cavitation

Compressibility

Shedding mechanism

OpenFOAM $^{\circledR}$

\begin{abstract}
A B S T R A C T
The periodic shedding of cloud cavitation has been previously assumed to be induced primarily by re-entrant jet based on considerable experimental and numerical studies. However, different shedding mechanisms, including that induced by pressure waves, have recently regained research interest. To conduct a corresponding numerical investigation, the cavitating flow around an axisymmetric projectile is studied using a user-designed solver that considers the compressibility of the three phases and phase change within the OpenFOAM ${ }^{\circledR}$ framework. Results are compared with those of an experimental study based on Split Hopkinson Pressure Bar (SHPB) technology with high-speed photography. Good agreement on cavity morphology is confirmed between the results. During the first period, a typical re-entrant jet-induced shedding mechanism is observed as the re-entrant jet front coincides with cavity closure. By contrast, their evident separation is noted in the second period, and cavity closure is located in a counterflow area caused by the impact of pressure waves that are radiated by the collapse of a shedding cavity and propagate in liquid water. This observation is never predicted by an incompressible solver used for comparison, thereby indicating that the existence of a different shedding mechanism is highly relevant to compressibility.
\end{abstract}

\section{Introduction}

The influence of cavitating flow is a concern in the design of many types of fluid machinery, such as hydrofoils, propellers, and pumps. A partial cavity exhibits violent instability under certain flow conditions and undergoes unsteady evolution, including periodic shedding and collapsing, which radiates pressure fluctuations and results in undesirable effects in engineering, such as erosion and noise. A shedding mechanism is essential to better estimate the relevant hydrodynamic performance to conduct further flow control. Knapp (1958) first proposed the re-entrant jet-induced shedding mechanism, which states that a high-velocity jet near wall is generated behind the cavity under a strong adverse pressure gradient and then moves upstream to cut off the cavity. Since then, many researchers have verified this finding using advanced experimental techniques (Kubota et al., 1989; Stutz and Legoupil, 2003; Callenaere et al., 2001). Cavitation models, including the single-fluid model and the transport equation model (TEM), have also been established (Kunz et al., 2000; Schnerr, 2001; Singhal et al., 2002), thereby promoting numerical studies on cavitation (Coutier-Delgosha et al., 2003; Huang et al., 2013; Ji et al., 2014).

However, the shedding mechanism is not solely attributed to reentrant jet. Many studies have reported two other types of shedding mechanism in cavitating flow. The first mechanism is due to the pressure waves generated by the extremely high pressure of cavity collapse, and these waves propagate in liquid water and later lead to residual cavity pinch-off. Arndt (2000) defined a characteristic parameter for cavitating flow around a hydrofoil to distinguish regimes governed by different shedding mechanisms. Song (2001) conducted numerical simulation using the single-fluid model for a hydrofoil. They found that the shedding cloud can break up into pieces, as opposed to the spherical cloud reported by Reisman (1998). Another interesting result is that a rarefaction will propagate to the pressure side of the hydrofoil, thereby resulting in considerable pressure decrease and the generation of a new cavity on that side. The second mechanism is a condensation shock that

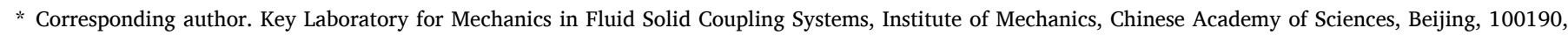
China.

E-mail address: wangyw@imech.ac.cn (Y. Wang).
} 
propagates through a cavity with phase change. This concept raised in general research on multiphase flow. Campbell and Pitcher (1958) investigated the propagation of shock in bubbly flow. On the basis of their work, Noordzij and Wijngaarden (1974) further considered the influence of the motion of bubbles. Brennen (1995) took into account phase change and found that acoustic velocity can be considerably less than that without phase change. Kawanami et al. (1997) observed condensation shock in experiments although they assumed that cavity shedding is caused by re-entrant jet. Reisman (1998) investigated the dynamics of the shedding cavity on a hydrofoil; they found that a condensation shock is generated on the surface of a spherical cloud and then propagates inward to cause collapsing. Ganesh et al. (2016) recently used X-Ray densitometry to detect the volume fraction in cavitating flow around a wedge, and they verified the shock-induced shedding mechanism. Following the former work, Budich et al. (2018) conducted numerical simulation with inviscid hypothesis and the single-fluid model. Wang et al. (2018) found that shock-induced shedding causes reduction of cavitation evolution Strouhal number on a hydrofoil. Moreover, the existence of both types of mechanism was also reported. Leroux et al. (2004) compared the results from both numerical simulations and experiments. They found that the collapse of a shedding cavity radiates pressure waves, which impinge on the weak of the residual and then cause it to collapse with a velocity that approximates the acoustic velocity in the bubbly mixture with phase change. In addition, the results showed that sheet cavity does not only exist at the 6 attack angle, which is also the required condition for the shock intensity to reach a maximum value. This finding suggests that cavity vanishing is associated with shock.

Numerical investigation among the aforementioned studies almost used the density-based compressible solver, which is originally designed for supersonic flows. The direct application of this type of solvers suffers from high stiffness and poor stability somewhere local Mach number is insufficiently high, e.g., in areas suffused by the liquid phase in cavitating flow. Thus, preconditioning technique has been established to extend its application to flow of all speeds (Turkel, 1987; Weiss and Smith, 1995; Bram et al., 1991). However, this approach is limited by deteriorating time precision when simulating unsteady flow. Venkateswaran and Merkle (1995) promoted dual time-stepping as an improvement; this approach was introduced by Kunz et al. (2000) to study cavitating flow. Although preconditioning can alleviate the difficulty of the density-based solver, physical compressibility is disregarded, thereby leading to the unsatisfactory resolution of pressure waves. Another choice is the pressure-based solver, which basically adopts prediction-correction algorithm. It is originally designed for incompressible flow, but has been extended it to flow at all speeds by series of researches (Issa and Javareshkian, 1998; Moukalled et al., 2003). Distinct advantage of this kind solver is good stability under any flow conditions. And it has also been applied in cavitating flow simulation (Senocak and Shyy, 2001).

At present, arguments remain on whether pressure waves or shocks is primary in shedding mechanism, and this issue requires further investigation. Previously incompressible solvers are widely used to simulate re-entrant jet. However, compressibility is required when studying collapse-induced pressure wave or shock propagation and its effect on cavitating flow. In the current work, a pressure-based compressible multiphase solver with cavitation phase change is established within the OpenFOAM $^{\circledR}$ framework, through which cavitating flow around an axisymmetric projectile is simulated with Large Eddy Simulation (LES). The profile and length of the cavity are first compared between the results obtained from the newly developed solver and experiments based on the Split Hopkinson Pressure Bar (SHPB) technology with high-speed photography. Then, the velocity and pressure fields in different stages predicted using the new solver are analyzed in detail to find the characteristic that distinguishes shedding induced by pressure waves or shocks from that induced by re-entrant jet. In addition, predictions are compared between both types of solvers in terms of the aforementioned issues.

\section{Methods}

\subsection{Governing equations}

As most references have indicated, the homogenous multiphase model, which disregards slip between phases and assumes that all the phases share the same velocity field, is adopted. Phase change between liquid water and vapor is introduced by the TEM category cavitation model. Define volume fraction of water and vapor as $\alpha_{1}, \alpha_{\mathrm{v}}$, respectively. Therefore, the phase transport equation for each phase of the mixture is as follows:

$$
\begin{aligned}
& \frac{\partial \alpha_{l}}{\partial t} \quad \nabla \cdot \alpha_{l} \mathbf{u} \quad \frac{\alpha_{l}}{\rho_{l}} \frac{D \rho_{l}}{D t} \quad \frac{\dot{m}}{\rho_{l}} \\
& \frac{\partial \alpha_{v}}{\partial t} \quad \nabla \cdot \alpha_{v} \mathbf{u} \quad \frac{\alpha_{v}}{\rho_{v}} \frac{D \rho_{v}}{D t} \quad \frac{\dot{m}}{\rho_{v}},
\end{aligned}
$$

where ${ }_{i}$ and $\boldsymbol{u}$, denote density and the velocity field shared by all the phases. Phase change rate $\dot{m}$ is the sum of evaporation rate and condensation rate that calculated by cavitation models, which is chosen as Kunz model in this study. The constraint $\alpha_{l} \quad \alpha_{v} \quad 1$ should be satisfied. These equations are derived by rearranging the continuity equation of each phase to provide convenience in solving processes.

The momentum equation for the mixture is as follows:

$$
\frac{\partial \rho \mathbf{u}}{\partial t} \quad \nabla \cdot \rho \mathbf{u u} \quad \nabla p \quad \nabla \cdot \mu \quad \nabla \mathbf{u} \quad \nabla \mathbf{u}^{\mathrm{T}} \quad \frac{2}{3} \nabla \cdot \mathbf{u} \mathbf{I} \quad \varsigma \kappa \nabla \alpha_{l},
$$

where the symbol $\rho$ without the subscript is the mixture density defined as $\rho \rho_{l} \alpha_{l} \quad \rho_{v} \alpha_{v}$. While $\mu, \varsigma, \kappa$ respectively denotes dynamic viscosity, surface tension coefficient and curvature. The last term on the right side represents the effect of surface tension force, which is active only within vicinity of the interfaces of liquid water and another phase. Gravity is disregarded in this study.

The energy equation for the mixture is

$\begin{array}{lllllll}\frac{\partial}{\partial t} \rho K & U & \nabla \cdot \rho \mathbf{u} K & U & \nabla \cdot \mathbf{u} \cdot \boldsymbol{\tau} & \nabla \cdot \mathbf{q} & \nabla \cdot p \mathbf{u},\end{array}$

where $U, \boldsymbol{q}$ denote specific internal energy and heat flux density, and kinetic energy $K \quad 1 / 2|\mathbf{u}|^{2}$.

For simplification, apart from disregarding the effect of shear stress, the following relations can be substituted into the energy equation:

$U \quad C_{v} T$,

q $\quad \alpha^{\text {th }} C_{v} \nabla T$,

Therefore, an equation for temperature $T$ can be derived as follows:

$$
\begin{array}{ccc}
\frac{\partial}{\partial t} \rho C_{v} T & \nabla \cdot \rho C_{v} T \boldsymbol{u} & \nabla \cdot \alpha^{\text {th }} C_{v} \nabla T \\
\nabla \cdot p \boldsymbol{u} & \frac{\partial}{\partial t} \rho K & \nabla \cdot \rho K \boldsymbol{u}
\end{array}
$$

where $C_{v}$ denotes the special heat capacity, and $\alpha^{\text {th }}$ is the thermal diffusivity.

\subsection{Numerical methods}

Besides good stability as mentioned above, pressure-based compressible solver is also convenient to be implemented in OpenFOAM $^{\mathbb{R}}$. Therefore, the solver established in current study belongs to this category. The phase transport equations are solved first within a time step. Only one of these equations need be solved. The volume fraction of the remaining phase, which is chosen as water in this study, is 
obtained through the constraint $\alpha_{l} \quad \alpha_{v} \quad$ 1. To solve these equations, the sharpness of the interface and the boundedness of the volume fractions should be ensured. OpenFOAM ${ }^{\circledR}$ provides an artificial compression term along with the multidimensional universal limiter with explicit solution (MULES) algorithm, which is based on the flux-corrected transport (FCT) method. Less precision is ensured than that of the standard volume of fluid method (VOF) while fewer computing resources are consumed. This process requires the phase transport equation of phase $i$ to be rearranged as follows:

$\frac{\partial \alpha_{i}}{\partial t} \quad \nabla \cdot \alpha_{i} \mathbf{u} \quad \nabla \cdot\left(\sum_{j} \alpha_{i} \alpha_{j} \mathbf{u}_{r i j}\right) \quad \frac{\alpha_{i}}{\rho_{i}} \frac{D \rho_{i}}{D t} \quad S$,

where the third term on the left hand side is the compression term, and $S$ denotes the source term of the phase change. $\boldsymbol{u}_{r i j}$ which represents interface velocity is estimated using the formula provided by Weller (2008):

$\mathbf{u}_{r i j} \quad \min c_{\alpha}|\mathbf{u}|, \max |\mathbf{u}| \frac{\nabla \alpha_{i}}{\left|\nabla \alpha_{i}\right|}$,

where $c_{\alpha}$ is used to adjust the sharpness of the interface, and its value is generally $0-2$.

The second and third terms on the left-hand side are discretized as

$\sum \alpha_{i} \mathbf{u}_{f} \cdot \mathbf{S}_{f} \quad \sum\left(\sum_{j} \alpha_{i} \alpha_{j} \mathbf{u}_{r i j}\right)_{f} \cdot \mathbf{S}_{f}$,

where $S_{f}$ is the area vector of control face. This formula should not be calculated directly and requires adjustment in accordance with the FCT method. Denote compression term $\sum F^{r}$ and convection term $\sum F^{L H}$, whose superscript indicates the use of a low(high)-order scheme. The original calculation is replaced by the following:

$\sum F^{L} \quad \lambda \sum F^{H} \quad \sum F^{r} \quad \sum F^{L}$,

where $\lambda$ is a coefficient defined in the FCT method.

The formulation of the pressure equation for compressible flows is also emphasized. Eq. (1) are summed up as

$\frac{\alpha_{l}}{\rho_{l}} \frac{D \rho_{l}}{D t} \quad \frac{\alpha_{v}}{\rho_{v}} \frac{D \rho_{v}}{D t} \quad \nabla \cdot \mathbf{u} \quad \frac{1}{\rho_{l}} \quad \frac{1}{\rho_{v}} \dot{m}$,

The discretized form is as follows:

$$
\begin{gathered}
\sum_{i} \frac{\alpha_{i}^{n} 1}{\rho_{i}^{\xi}} \frac{\partial \rho_{i}^{\xi}}{\partial t} \Delta V \quad \sum \rho_{i}^{\xi} \boldsymbol{u}_{f}^{\xi} \cdot S_{f} \quad \rho_{i}^{\xi} \sum \boldsymbol{u}_{f}^{\xi} \cdot \boldsymbol{S}_{f} \quad \sum \boldsymbol{u}_{f}^{\xi}{ }^{1} \cdot \boldsymbol{S}_{f} \\
\frac{1}{\rho_{l}^{\xi}} \frac{1}{\rho_{v}^{\xi}} \dot{m}
\end{gathered}
$$

where the superscripts $n$ and $\xi$ respectively denote the time step and prediction-correction step. The time derivative term can be replaced by any temporal scheme. If $\boldsymbol{u}^{\xi} 1$ and $\rho$, which can be derived from the momentum and equation of state, are substituted, then an unsteady convection-diffusion pressure equation is obtained.

\subsection{Turbulence modeling}

A turbulence model is crucial for the numerical simulation of cavitating flow because of its natural unsteadiness. Simulations based on Reynolds-averaged Navier-Stokes (RANS) have been widely applied and recognized in engineering. However, many recent studies indicate that LES can capture considerable details of small-scale flow structures in cavitating flow and provide better predictions of large-scale turbulent eddies with higher accuracy (Orley et al., 2015; Chen et al., 2019). To perform LES, Favre filtering is taken since variation of density is considered. And implicit filtering is applied as implemented in
OpenFOAM ${ }^{\circledR}$. The momentum and temperature equations are filtered as:

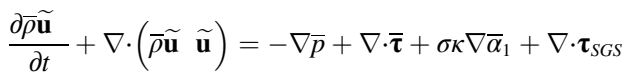

$$
\begin{array}{rllllll}
\frac{\partial}{\partial t} \bar{\rho} C_{v} \tilde{T} & \nabla \cdot \bar{\rho} C_{v} \tilde{T} \tilde{\boldsymbol{u}} & \frac{\partial}{\partial t} \bar{\rho} \frac{\tilde{\boldsymbol{u}} \cdot \tilde{\boldsymbol{u}}}{2} & \nabla \cdot \bar{\rho} \frac{\tilde{\boldsymbol{u}} \cdot \tilde{\boldsymbol{u}}}{2} \tilde{\boldsymbol{u}} & \\
& \nabla \cdot \alpha^{t h} C_{v} \nabla \bar{T} & \nabla \cdot \bar{p} \tilde{\boldsymbol{u}} & \nabla \cdot \boldsymbol{Q}_{S G S}
\end{array}
$$

where the tilde quantities are Favre averaged ones. $\boldsymbol{\tau}_{S G S}, \boldsymbol{Q}_{S G S}$ are subgrid scale (SGS) stress and SGS heat flux that are brought by nonlinear in governing equations and need to be modelled.

The one-equation eddy-viscosity model is adopted in the present study for SGS stress modeling. Compared with Smagorinsky model, this type of filter exhibits the advantage of providing a more accurate time scale through the independent definition of the velocity scale and has demonstrated advantages when used in modeling transitional flows or flows with large-scale unsteadiness (Villiers, 2006). Its SGS eddy-viscosity $\nu_{S G S}$ and dissipation $\varepsilon$ are defined as

$\nu_{S G S} \quad C_{k} \frac{k_{S G S}^{1 / 2}}{\Delta}$,

$\varepsilon \quad C_{\varepsilon} \frac{k_{S G S}^{3 / 2}}{\Delta}$.

where $\Delta$ denotes filter width that is chosen as cube-root of the cell volume in this study, and coefficients are given as $C_{k} 0.094$, $C_{\varepsilon}$ 1.048. The SGS kinetic energy $k_{S G S}$ is solved through its transport equation:

$\begin{array}{cccc}\frac{\partial \bar{\rho} k_{S G S}}{\partial t} & \nabla \cdot \bar{\rho} \tilde{\boldsymbol{u}} k_{S G S} & \nabla \cdot \bar{\rho} \nu & \nu_{S G S} \nabla k_{S G S} \\ \bar{\rho} \boldsymbol{\tau}_{S G S}: \overline{\boldsymbol{D}} & \bar{\rho} \varepsilon\end{array}$

where $\overline{\mathbf{D}}$ is the resolved-scale strain rate tensor. And the SGS stress tensor $\tau_{i j}$ is modelled as

$\boldsymbol{\tau}_{S G S} \quad \frac{2}{3} k_{S G S} \mathbf{I} \quad 2 \nu_{S G S} \quad \overline{\mathbf{D}} \quad \frac{1}{3} \operatorname{tr} \overline{\mathbf{D}} \mathbf{I}$

The SGS heat flux are simply modelled as a diffusion term:

$\mathbf{Q}_{S G S} \frac{\bar{\rho} \nu_{S G S}}{\operatorname{Pr}_{S G S}} \nabla T^{\sim}$

where $\operatorname{Pr}_{\text {SGS }}$ is SGS Prandtl number, chosen as 1.0 in internal computational domain and 0.85 at walls.

\subsection{Performance test for the solver}

Since the solver is newly developed, it is crucial to conform whether it can correctly capture unsteady cavitating flow. Therefore, a simple case about 2D cavitating flow around a circular cylinder is chosen to carry out performance test. Reynolds number Re and cavitation number $\sigma$ in this test is respectively set as 200 and 1.0, at which values flow remains laminar and strong regularity of the flow field should be observed.

Fig. 1(a) shows evolution of cavity shape within a typical cycle. The flow is found to be strongly periodic as cavity sheds alternately on each side. A cavity on one side grows rounded at first, being gradually stretched later. When it almost sheds from the cylinder, the cavity on the other sides begins to grow, which marks the end of first half cycle. Fig. 1 (b) shows diagram of lift/drag coefficient vs. time. Like the situation in non-cavitating flow, lift coefficient is half the frequency of drag coefficient. But by contrast, the curves become much steeper near each extreme point when cavitation happens. These results agree with Gnanaskandan and Mahesh (2016), which indicates that the solver is 
(a)

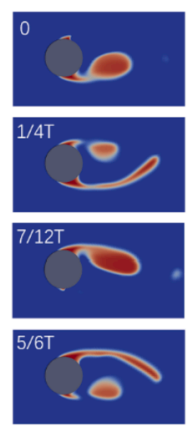

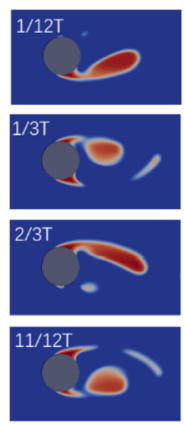

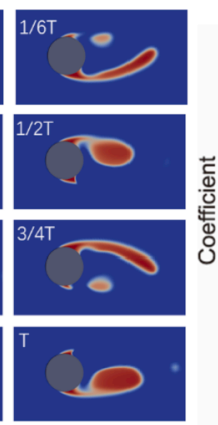

(b)

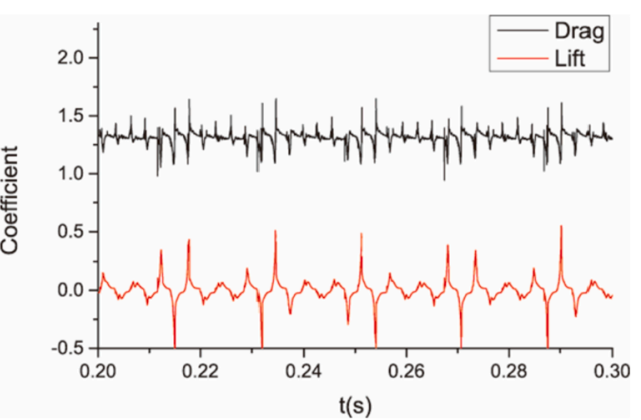

Fig. 1. Results of 2D cavitating flow around a circular cylinder; (a) evolution of cavity shape expressed by counter of volume fraction where red areas denote cavity; (b) evolution of lift and drag coefficients vs. time.

qualified in current test.

\subsection{Simulation setup}

The newly established solver is used to study cavitating flow around an axisymmetric projectile with emphasis on the shedding mechanism. A cylindrical computational domain with a structured mesh is adopted as shown in Fig. 2. The diameter of the projectile $d$ is $37 \mathrm{~mm}$, which is equivalent to the height of the conical head. Gravity is disregarded because of the small geometric scale. Inflow velocity is $18.5 \mathrm{~m} / \mathrm{s}$, which indicates a moderate cavitation number $\sigma \quad 0.57$. The boundary conditions of several selected variables are provided in Table 1 . To prevent the reflection of pressure waves onto outside boundaries, a boundary condition named waveTransmissive is applied for $\mathrm{p}$, thereby requiring other variables to adopt the corresponding settings.

The setup of this case is completely based on previous works ( $\mathrm{Yu}$ et al., 2014; Wang et al., 2016), where grid convergence study was taken. Thus, mesh of equivalent density and topology is adopted. And in their experiment, a projectile was launched in a tank using the SHPB technology, and the entire process is recorded via high-speed photography. The results show the rapid vanishing of the residual cavity, which is considered nontypical shedding caused by re-entrant jet and may be caused by pressure waves or shocks. Thus, the results provided by the new solver are compared with the experimental results to verify the aforementioned conjecture. By contrast, the interPhaseChangeFoam, which is previously widely used in cavitation problems as an incompressible solver originally contained in OpenFOAM ${ }^{\circledR}$, is launched for a

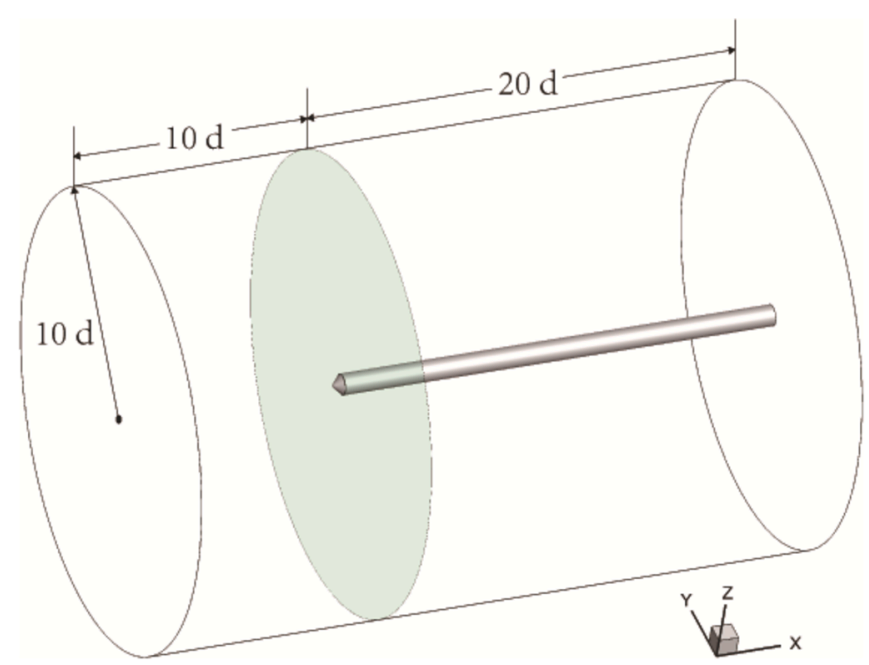

Fig. 2. Computational domain, where d denote diameter of the projectile and its value is $37 \mathrm{~mm}$.
Table 1

Boundary conditions of some selected variables.

\begin{tabular}{|c|c|c|c|c|}
\hline variables & Inlet & Outlet & Projectile & Sides \\
\hline alphai & fixed value & zero gradient & $\begin{array}{l}\text { zero } \\
\text { gradient }\end{array}$ & fixed value \\
\hline $\mathrm{p}$ & $\begin{array}{l}\text { pressure wave } \\
\text { transmissive }\end{array}$ & fixed value & $\begin{array}{l}\text { zero } \\
\text { gradient }\end{array}$ & $\begin{array}{l}\text { pressure wave } \\
\text { transmissive }\end{array}$ \\
\hline $\mathrm{U}$ & $\begin{array}{l}\text { fixed value with } \\
\text { perturbation }\end{array}$ & $\begin{array}{l}\text { zero gradient } \\
\text { with backflow }\end{array}$ & fixed value & zero gradient \\
\hline $\mathrm{k}$ & $\begin{array}{l}\text { fixed value with } \\
\text { perturbation }\end{array}$ & zero gradient & $\begin{array}{l}\text { zero } \\
\text { gradient }\end{array}$ & zero gradient \\
\hline nut & unnecessary & unnecessary & $\begin{array}{l}\text { using wall } \\
\text { function }\end{array}$ & unnecessary \\
\hline $\mathrm{T}$ & fixed value & zero gradient & fixed value & zero gradient \\
\hline
\end{tabular}

comparative study. If pressure waves or shock waves exist physically, then this treatment will be unreasonable for its elliptical system. Nevertheless, the potential incorrect usage of this solver can be presented in such a manner.

\section{Results and discussion}

\subsection{Comparison with experimental results}

Fig. 3 shows a brief comparison of the cavity profile within a certain period to validate evolution of cavity shape. A cavity grows from shoulderpoint of a projectile, originally as a transparent sheet cavity. When its length is near maximum, re-entry jet is generated, making the cavity cloudy from its closure and going upstream to cut off the cavity, which corresponds to the inner profile in the simulation results. Soon afterwards a new cavity appears on the shoulder point, whereas the shedding part moves downstream and collapses later. The main residual cavity attached to the projectile then remains with the fragmentary parts downstream and begins to shed. Considerable agreement on cavity shape is validated in the majority of a period except for the fragmentary cavities downstream, which are thicker as observed in the experiment.

To make a quantitative validation, dimensionless cavity length $L^{*} \quad L / d$ is plotted versus dimensionless time $T^{*} \quad t / T$ in Fig. 4, where $L$, $d$ and $T$ denotes cavity length, projectile diameter, and period, respectively. Cavity length is defined as the horizontal distance between the shoulder point and the most downstream point of a cavity, disregarding several instances of breaking up midstream. The trend of both results is accordant and the entire period can be divided into four stages: a rapid original increase, a slow decrease, another increase with subequal slope, and a final abrupt decrease to a non-zero value. These stages physically represent cavity growth, re-entry jet generation and cavity shedding, new cavity growth, and collapse of shedding cavities and pressure wave or shock propagation. Although the maximum values of $L^{*}$ are in good agreement, several differences remain. In Stage $1, L$ *obtained from the 


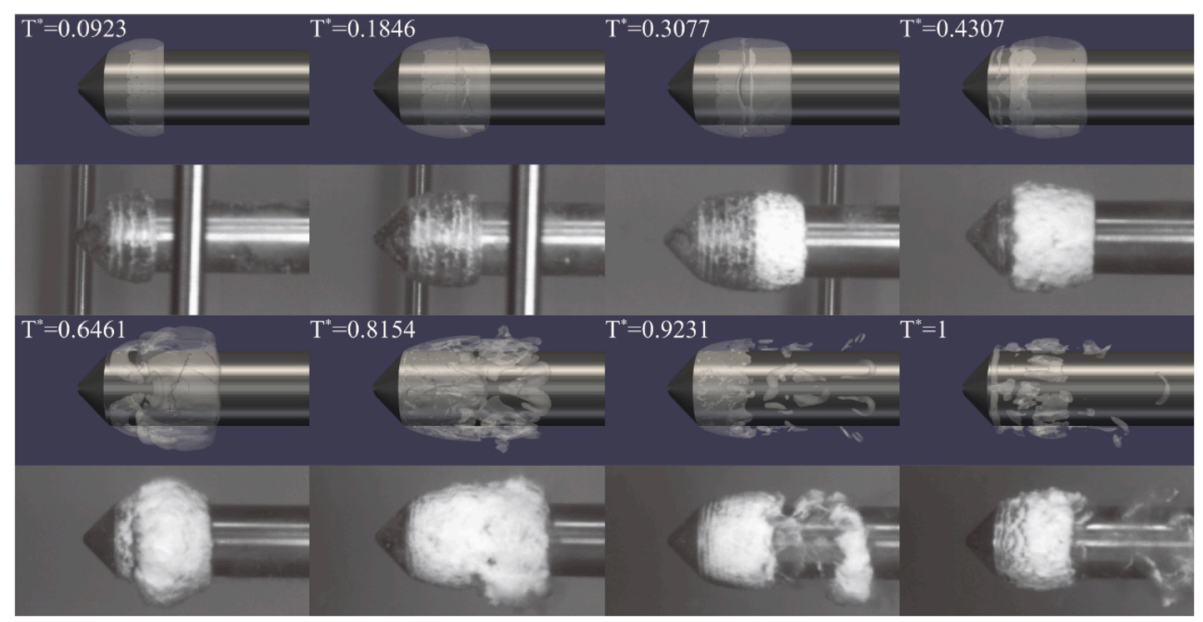

Fig. 3. Morphology comparison of the cavity between numerical results and experiment in one period.

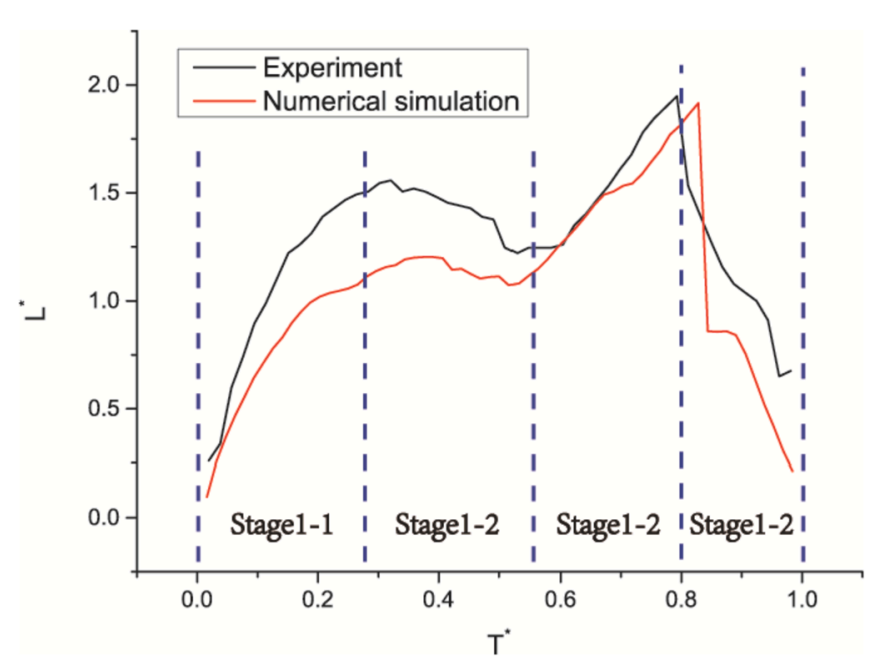

Fig. 4. Comparison of dimensionless cavity length $L^{*}$ vs. dimensionless time $T^{*}$ in one period.

experiment is larger than the numerical prediction. In Stage 2-2, the numerical results experience considerably more fierce falling, indicating that the cavity vanishes immediately.

\subsection{Shedding induced by re-entrant jet in stage 1}

The evolution of the streamwise velocity and pressure fields, along with the cavity profile in Stage 1, is briefly shown in Fig. 5. The cavity profile is extracted with a volume fraction of vapor $\alpha_{2} \quad 0.3$. As the cavity grows in the beginning, flow separation occurs, along with the adverse pressure gradient near the cavity closure. When the maximum cavity length is nearly reached, a thin layer of liquid near the wall begins to move upstream and is gradually accelerated, thereby forming a typical re-entrant jet, as shown by the dark blue area in the streamwise velocity field. This area continuously extends upstream and finally reaches the vicinity of the shoulder point, which leads to breaking up of the cavity profile and marks the end of Stage 1 . This process is a typical re-entrant jet-induced shedding depicted in many studies. It is reviewed here with an emphasis on its characteristic, i.e., the front of re-entrant jet is exactly coincident with the inner profile of the cavity as shown in the contour of streamwise velocity. Furthermore, no pressure wave nor shock propagation is observed during this stage without evident cavity collapse and cavity profile remains nearly smooth.

Cavity evolution in Stage $2-1$ is shown in Fig. 6. As a new cavity grows on the shoulder point, the shedding part breaks up into fragments and merges later, connecting to the newly generated part. The new coalescent cavity rotates downstream, becoming thicker first and then shrinking later. Velocity is lower inside the profile of the cavity than outside it, thereby indicating that the streamwise motion of the cavity is slower than the free stream. When cavity length is near the maximum value, pressure on cavity closure is beyond the saturated pressure and collapse begins to occur, which leads to local high pressure. This process implies the end of Stage $2-1$. Interestingly, when the cavity is growing, flow separation covered by the cavity has existed, and this area moves downstream, thereby developing to re-entrant jet prior to cavity collapse.

\subsection{Pressure wave-induced shedding in stage 2-2}

The results of the aforementioned stages indicate that the newly developed solver does not predict considerably more than previous studies. However, substantial attention should be given to Stage 2-2 when a new phenomenon is found.

The tail of the cavity suddenly experiences large-scale cavity collapse, which corresponds to abruptly decreasing cavity length, as shown in Fig. 4. The main part of the residual cavity remains attached to the projectile, with fragmentary parts located on the horseshoe vortex moving slowly downstream or collapsing locally. Then, a high-pressure center of up to $0.6 \mathrm{MPa}$ is generated due to the large-scale collapse, and the propagation of the radiated pressure waves in liquid water is detected, as illustrated by the contour of the pressure gradient with the cavity profile in Fig. 7. As the residual cavity is swept over by pressure waves, an isolated high-pressure center evidently appears on its closure, resulting in local secondary collapse. It is remarkable that no wave patterns are found in the cavity profile, which is expected becausea considerable difference in acoustic impedance between liquid water and vapor leads to sharp attenuation on both wave energy and amplitude after a wave propagates through the interface of two phases.

The streamwise velocity field, along with the cavity profile countered by the condensation rate, is shown in Fig. 8. When the tail of the cavity collapses at $T^{*} \quad 0.8154$, the re-entrant jet is not simultaneously extended upstream, and therefore, a notable separation appears between its front and the closure of the residual cavity. This situation significantly differs from the situation in Stage 1, which is characterized by the coincidence of the two components. However, the cavity length of the residual cavity continues to decrease, thereby indicating that the reentrant jet is not the primary reason for the vanishing of the cavity. Instead, a counterflow velocity area is formed on its closure, which is generated by the impact of pressure waves at $T^{*} \quad 0.8154-0.8307$. Moreover, a secondary re-entrant jet can be found at $T^{*} \quad 0.8769$, which 


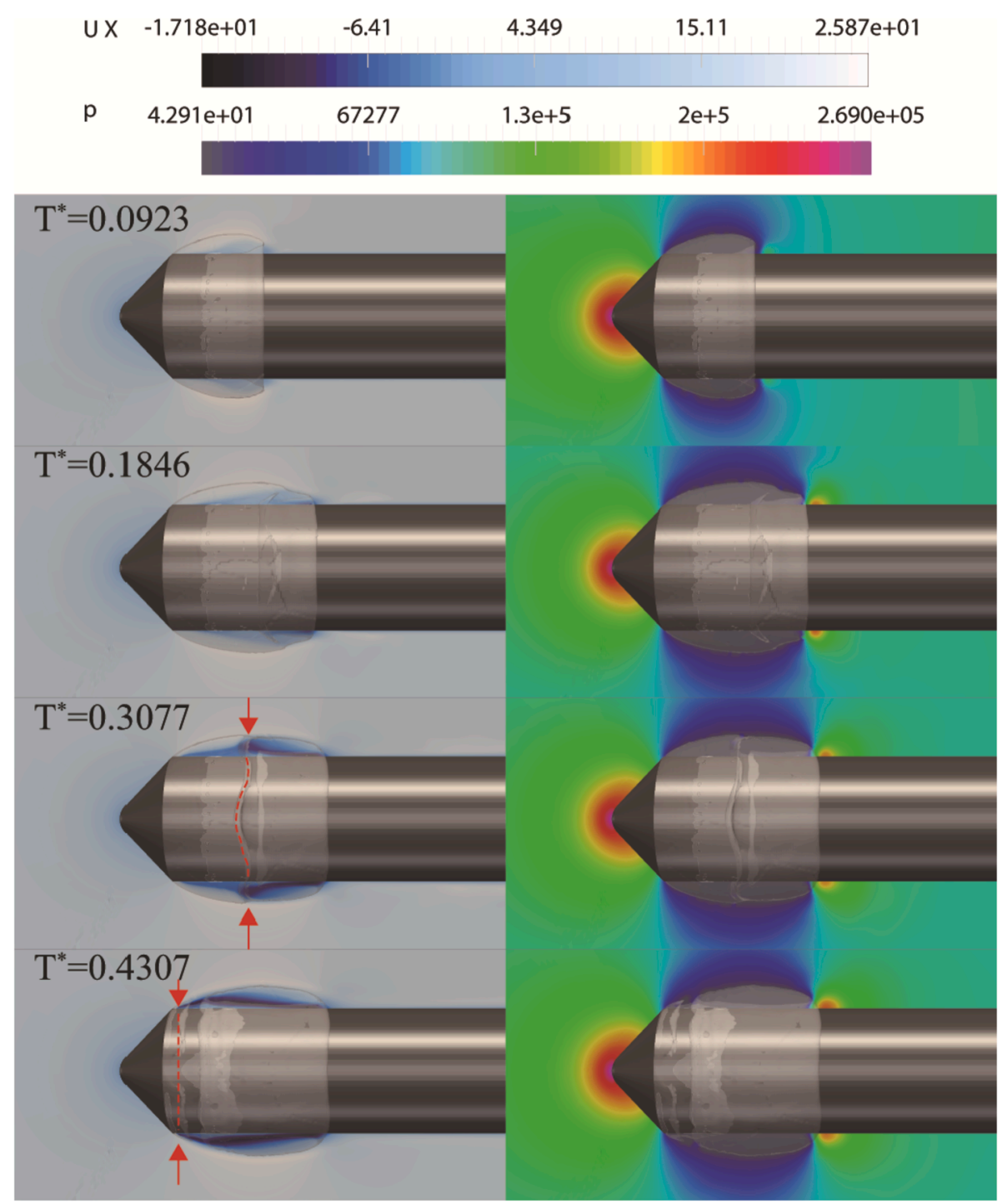

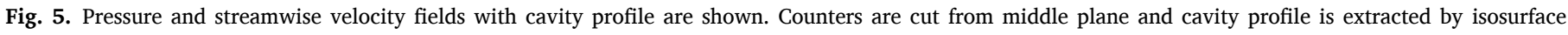
$\alpha_{2} \quad$ 0.3. The arrows and dash lines indicate the inner profile of a cavity, which is coincident with the front of re-entrant jet.

is also separated from the main re-entrant jet and promotes cavity shedding. Separation nearly exists to the end of this stage; thus, it can be regarded as evidence of the existence of a different shedding mechanism.

As introduced above, two types of shedding mechanisms exist in cavitating flow except re-entrant jet, i.e., collapse-induced pressure waves that only propagate in liquid water and condensation shock that propagates in mixtures with phase change. Leroux et al. (2004) investigated cavitating flow around a hydrofoil and reported that a condensation shock was found after the partial cavity is impacted by a collapse-induced pressure wave. However, the former type is not observed in this study because the cavity closure, which is directly impacted by pressure waves, does not exhibit a sufficiently high condensation rate high enough, and on the other hand pressure gradient field inside the residual cavity does not present any pressure wave. In conclusion, the impact of the collapse-induced pressure waves can be a trigger for continuously vanishing of a cavity, but not directly cause shedding while simultaneously propagating inside.

On the other hand, it is known that phenomenon in cavitating flow can be different as cavitation number $\sigma$ varies. For current study, it needs further investigation whether the pressure induced shedding mechanism that presents at $\sigma \quad 0.57$ as analyzed in preceding sections can still be observed with other values of $\sigma$. For this reason, cases with $\sigma \quad 0.9,1.1$ are carried out via the new solver, since larger $\sigma$ values will potentially eliminate influence of pressure waves. It is found that larger cavity length maximum and shorter cycle are predicted as $\sigma$ value increases. Other corresponding results are shown in Fig. 9, indicating the cavity shape with streamwise velocity in Stage 2-2. In both working conditions, the thick re-entrant jet is generated and strips the tail of residual cavity from the projectile. Moreover, its front is still found to separate from cavity closure as the situation at $\sigma \quad 0.57$. Thus, it is indicated that pressure wave induced shedding mechanism essentially exists in cavitating flow around the conical-head axisymmetric projectile. And this shedding mechanism, as appearing in Stage 2-2, is not changed by $\sigma$ value variation.

\subsection{Comparison with incompressible solver}

Analysis above indicates that quick vanishing of a residual cavity is strongly related to the impact of pressure waves. Investigating the behavior of incompressible solvers that are based on the elliptic system with infinite acoustic speed is meaningful. Therefore, interPhaseChangeFoam, an incompressible solver that is originally contained in OpenFOAM ${ }^{\circledR}$, is used to launch the case. The results are shown in 


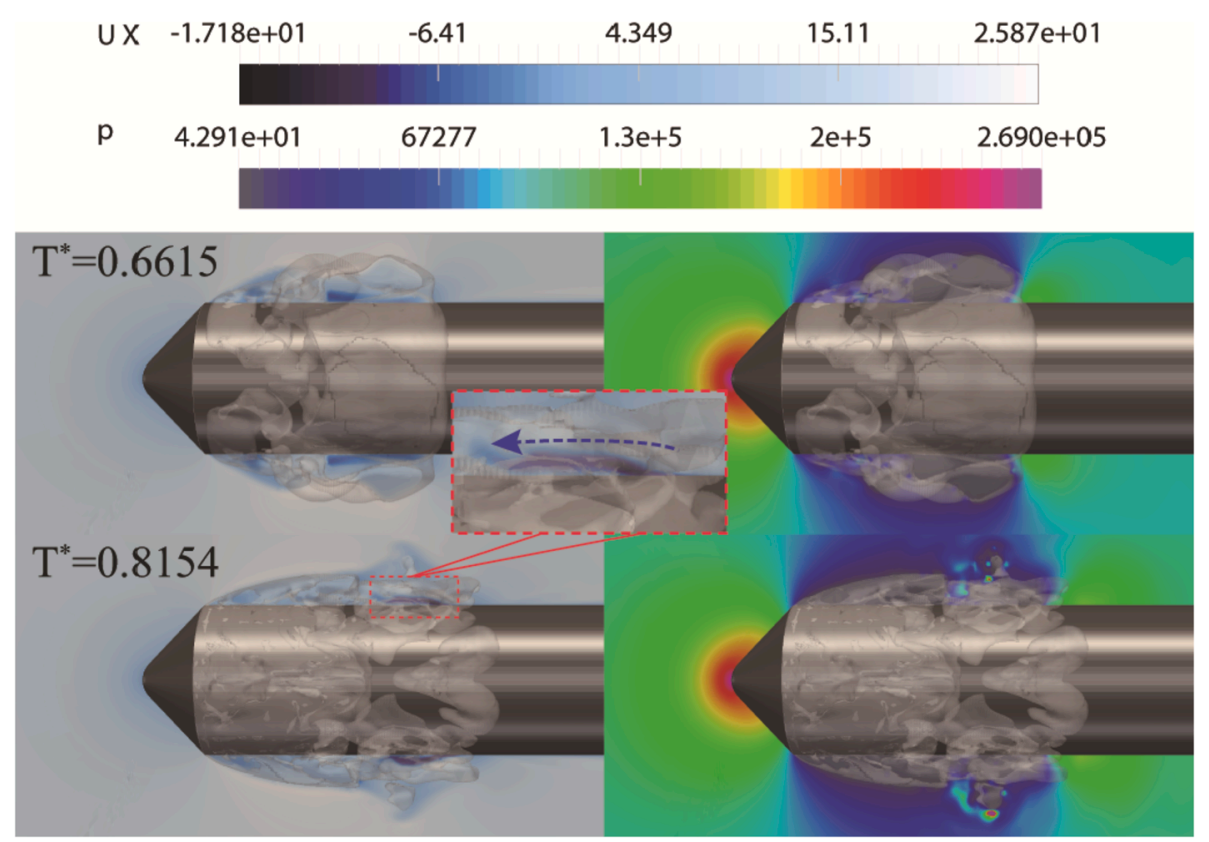

Fig. 6. Cavity evolution in Stage 2-1. A re-entrant jet has been generated before large-scale collapse occurs. Local magnification shows the generated re-entrant jet.

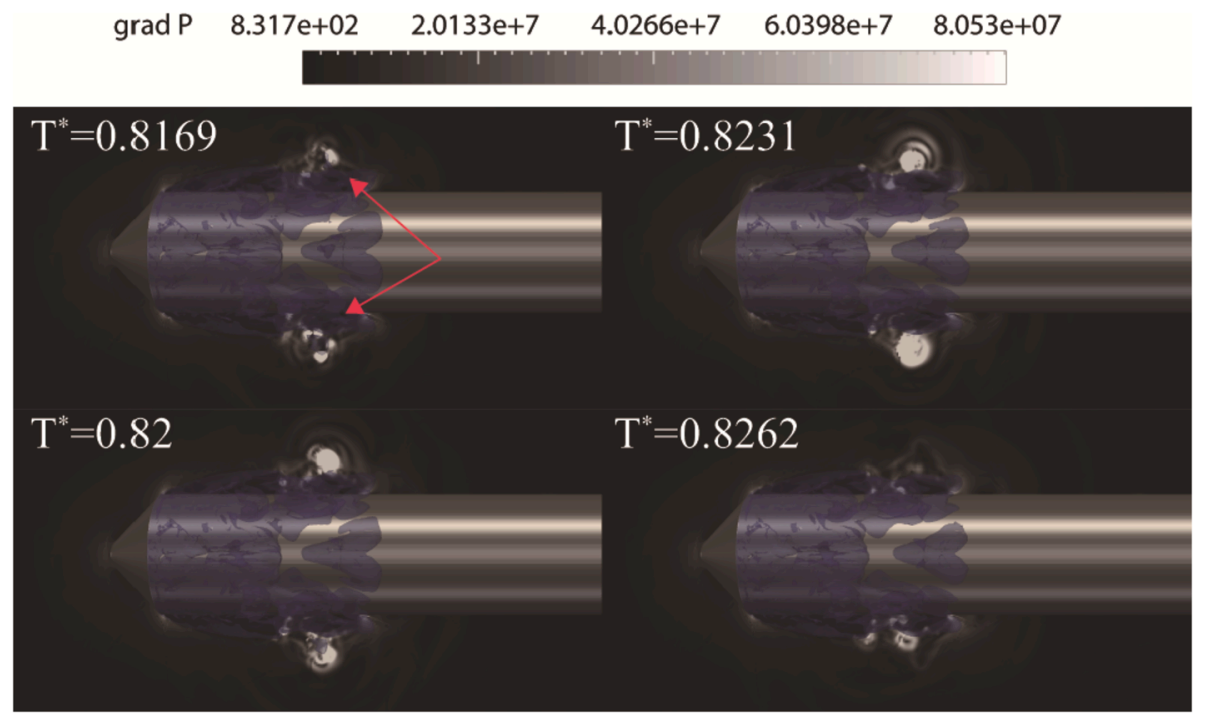

Fig. 7. Pressure wave propagation shown in the counter of the pressure gradient. The area shown by the arrows exhibits evident pressure waves.

Fig. 10. Compared with the situation in the compressible solver, only a few differences for the shedding progress are found before Stage 2-2 because no collapse-induced pressure wave propagation occurs, and re-entrant jet-induced shedding mechanism is naturally dominant. From Stage 2-2, however, a thick re-entrant jet is predicted by the incompressible solver, and its front remains coincident with the profile of the residual cavity, which is reasonably incorrect and is similar to typical reentrant jet-induced shedding. This result does not agree with vanishing of cavity under the pressure wave impact predicted by the new solver. Thus, the prediction of incompressible solvers can be reliable results in certain stages, but several important characteristics are lost when largescale collapse occurs.

\section{Conclusion}

In this study, a new compressible solver that considers three phases and the phase change between liquid water and vapor is established within the OpenFOAM ${ }^{\circledR}$ framework Based on this solver, cavitating flow around an axisymmetric projectile is studied through LES simulation with an emphasis on shedding mechanism.

The numerical results are first compared with those from the experiment and good agreement on morphology is confirmed. The diagram of dimensionless cavity length $L / D$ versus dimensionless time $t / T$ is also compared for validation, and four stages are defined for analysis. In Stage 1, the re-entrant jet-induced shedding mechanism is found to be characterized by a coincidence between cavity closure and the front of re-entrant jet. In Stage 2-1, re-entrant jet is observed to be generated prior to cavity collapse. In Stage 2-2, the pressure wave-induced shedding mechanism is found. Rapid vanishing of a cavity is due to a pressure wave, which is radiated by large-scale cavity collapsing downstream and propagates in liquid water. Thus, residual cavity closure is impacted and a counterflow area is generated. This shedding mechanism is characterized by the separation between re-entrant front and cavity closure and it is not changed by cavitation number variation. 

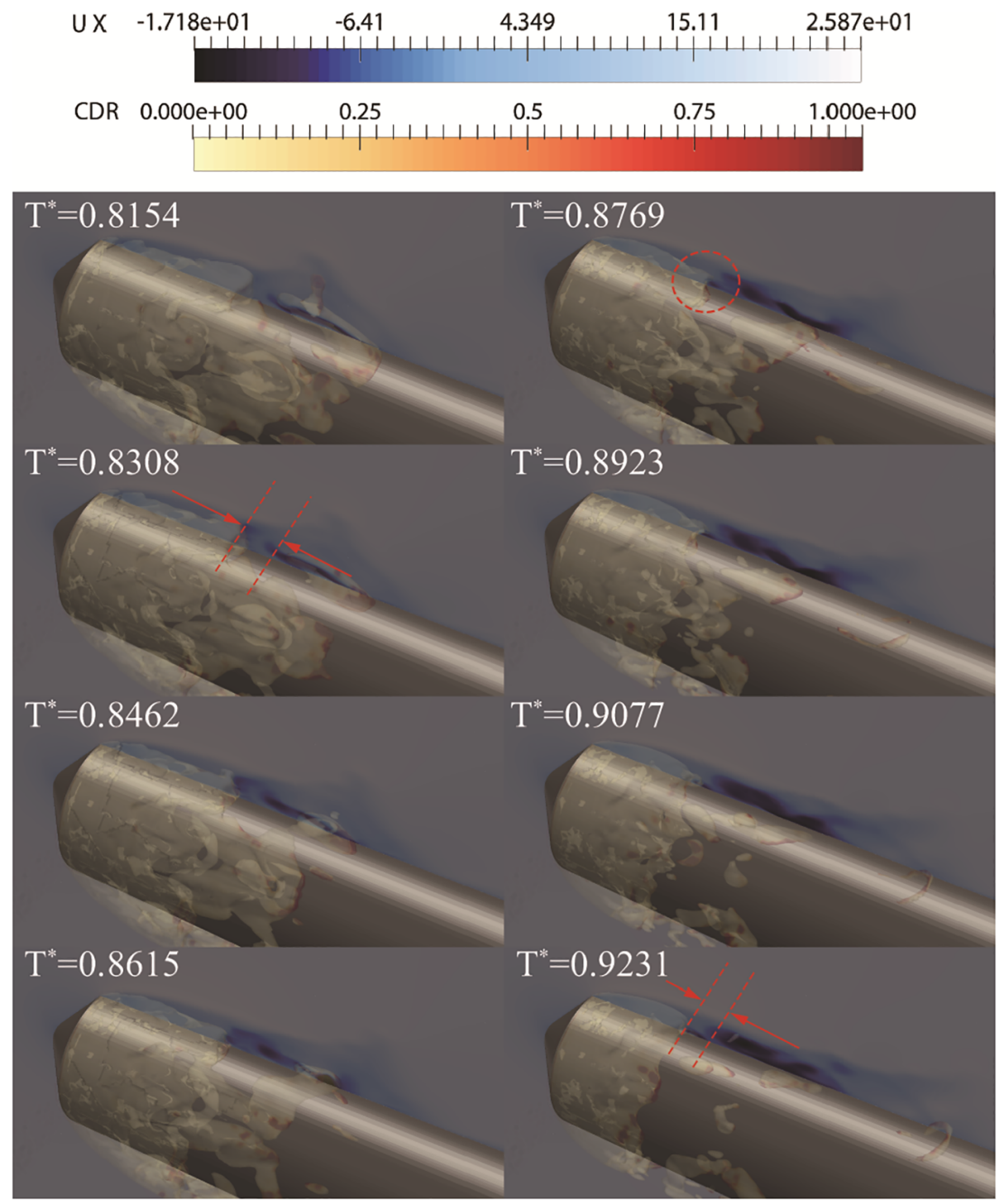

Fig. 8. High condensation rate is represented on as the red region on the translucent cavity profile (isosurface $\alpha_{2} \quad 0.3$ ). The lines and arrows show the separation between cavity closure and front of re-entrant jet in lattices $T^{*} \quad 0.8307$ and $T^{*} \quad 0.9231$. The circle in lattice $T^{*} \quad 0.8769$ points out the secondary re-entrant jet.

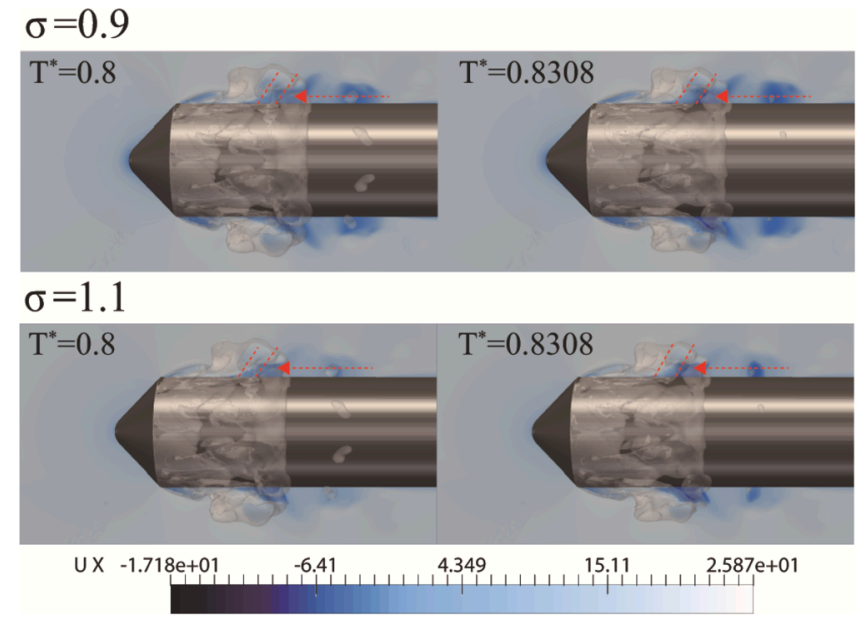

Fig. 9. Streamwise velocity field with cavity profile in Stage 2-2 when working condition is chosen as $\sigma \quad 0.9,1.1$. Re-entrant jet front is still found to separate from cavity closure.

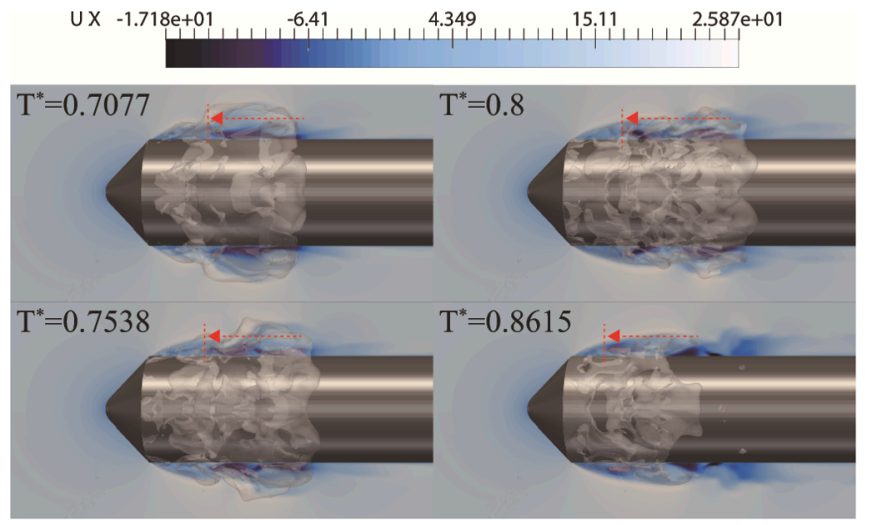

Fig. 10. Streamwise velocity field with cavity profile is shown. The lattice of $T^{*} \quad 0.7077$ is in Stage 2-1, and the remaining lattices are in Stage 2-2.

This case is also launched by interPhaseChangeFoam to investigate the behavior of incompressible solvers after cavity collapse. A coincidence between the front of re-entrant jet and cavity closure still appears in Stage 2-2, indicating that a typical re-entrant jet-induced shedding 
mechanism is predicted by this type of solvers. This result differs from that predicted by the newly developed solver and is considered to lose important characteristics. Therefore, employment of incompressible solvers in such a case is unsuitable.

The shedding mechanism is an important issue and needs further study. This study presents only one working condition for a certain model in cavitating flow. More investigation needs conduction to depict flow regime for shedding mechanism detailly. For more applications, the solver presented in this study is expected to extended with a phase of non-condensable gas, since multiphase cavitating flow has received increasing attention as a complex problem in engineering practice. Besides considering effect of non-condensable gas injection on cavity dynamics under current study, it will inspire many other investigations like water entry problems or free surface-piercing object where cavitating flow with cavitation happens.

\section{Acknowledgment}

This work was supported by the National Natural Science Foundation of China, grant numbers 11772340, and 11672315, the Youth Innovation Promotion Association CAS (2015015).

\section{References}

Arndt, R.E.A., 2000. Instability of partial cavitation: a numerical/experimental approach. In: Proceedings 23rd Symposium on Naval Hydrodynamics. Val de Reuil, France.

Bram, V.A., Lee, W., Roe, P., 1991. Characteristic time-stepping or local preconditioning of the Euler equations. In: 10th Computational Fluid Dynamics Conference, Honolulu,HI,U.S.A, pp. 260-282.

Brennen, C.E., 1995. Cavitation and Bubble Dynamics. Oxford University Press, New York.

Budich, B., Schmidt, S.J., Adams, N.A., 2018. Numerical simulation and analysis of condensation shocks in cavitating flow. J. Fluid Mech. 838, 759-813.

Callenaere, M., Franc, J.P., Michel, J.M., Riondet, M., 2001. The cavitation instability induced by the development of a re-entrant jet. J. Fluid Mech. 444, 223-256.

Campbell, I.J., Pitcher, A.S., 1958. Shock waves in a liquid containing gas bubbles. Proc. R. Soc. Lond. 243 (1235), 534-545.

Chen, Y., Li, J., Gong, Z., Chen, X., Lu, C., 2019. Large eddy simulation and investigation on the laminar-turbulent transition and turbulence-cavitation interaction in the cavitating flow around hydrofoil. Int. J. Multiph. Flow 300-322.

Coutier-Delgosha, O., Reboud, J.L., Delannoy, Y., 2003. Numerical simulation of the unsteady behaviour of cavitating flows. Int. J. Numer. Methods Fluids 42 (5), 527-548.

Ganesh, H., Makiharju, S.A., Ceccio, S.L., 2016. Bubbly shock propagation as a mechanism for sheet-to-cloud transition of partial cavities. J. Fluid Mech. 802, $37-78$.

Gnanaskandan, A., Mahesh, K., 2016. Numerical investigation of near-wake characteristics of cavitating flow over a circular cylinder. J. Fluid Mech. 790, 453-491.

Huang, B., Young, Y.L., Wang, G.Y., Shyy, W., 2013. Combined experimental and computational investigation of unsteady structure of sheet/cloud cavitation. J. Fluids Eng. Transac. ASME 135 (7), 16.

Issa, R.I., Javareshkian, M.H., 1998. Pressure-based compressible calculation method utilizing total variation diminishing schemes. AIAA J. 36 (9), 1652-1657.
Ji, B., Luo, X.W., Arndt, R.E.A., Wu, Y.L., 2014. Numerical simulation of three dimensional cavitation shedding dynamics with special emphasis on cavitationvortex interaction. Ocean Eng. 87, 64-77.

Kawanami, Y., Kato, H., Yamaguchi, H., Tanimura, M., Tagaya, Y., 1997. Mechanism and control of cloud cavitation. J. Fluids Eng. 236 (4), 788-794.

Knapp, R.T., 1958. Recent investigations of the mechanics of cavitation and cavitation damage. Oct. 1955 Robert T. Knapp. ASME, transactions 77, 1045-1054. Wear 1 (5), 455.

Kubota, A., Kato, H., Yamaguchi, H., Maeda, M., 1989. Unsteady structure measurement of cloud cavitation on a foil section using conditional sampling technique. J. Fluids Eng. Transac. ASME 111 (2), 204-210.

Kunz, R.F., Boger, D.A., Stinebring, D.R., Chyczewski, T.S., Lindau, J.W., Gibeling, H.J., Venkateswaran, S., Govindan, T.R., 2000. A preconditioned Navier-Stokes method for two-phase flows with application to cavitation prediction. Comput. Fluid 29 (8), 849-875.

Leroux, J.-B., Astolfi, J.A., Billard, J.Y., 2004. An experimental study of unsteady partial cavitation. J. Fluids Eng. Transac. ASME 126 (1), 94-101.

Moukalled, F., Darwish, M., Sekar, B., 2003. A pressure-based algorithm for multi-phase flow at all speeds. J. Comput. Phys. 190 (2), 550-571.

Noordzij, L., Wijngaarden, L.V., 1974. Relaxation effects, caused by relative motion, on shock waves in gas-bubble/liquid mixtures. J. Fluid Mech. 66 (1), 115-143.

Orley, F., Trummler, T., Hickel, S., Mihatsch, M.S., Schmidt, S.J., Adams, N.A., 2015. Large-eddy simulation of cavitating nozzle flow and primary jet break-up. Phys. Fluids 27 (8).

Reisman, G.E., 1998. Observations of shock waves in cloud cavitation. J. Fluid Mech. 355 (01), 255-283.

Schnerr, 2001. Physical and numerical modeling of unsteady cavitation dynamics. In: 4th International Conference on Multiphase Flow, New Orleans, LA, pp. 1-12.

Senocak, I., Shyy, W., 2001. Numerical Simulation of Turbulent Flows with Sheet Cavitation.

Singhal, A.K., Athavale, M.M., Li, H.Y., Jiang, Y., 2002. Mathematical basis and validation of the full cavitation model. J. Fluids Eng. Transac. ASME 124 (3), 617-624.

Song, C.C.S., 2001. Numerical simulation of unsteady cavitating flow. In: Proc. Of the Fourth International Symposium on Cavitation, Pasadena, California.

Stutz, B., Legoupil, S., 2003. X-ray measurements within unsteady cavitation. Exp. Fluid 35 (2), 130-138.

Turkel, E., 1987. Preconditioned methods for solving the incompressible and low-speed compressible equations. J. Comput. Phys. 72 (2), 277-298.

Venkateswaran, S., Merkle, C., 1995. Dual time-stepping and preconditioning for unsteady computations. In: 33rd Aerospace Sciences Meeting and Exhibit, Reno, NV, USA, p. 78.

Villiers, E.d., 2006. The Potential of Large Eddy Simulation for the Modelling of Wall Bounded Flows. Department of Mechanical Engineering. Imperial College London, p. $66 \sim 67$.

Wang, C.C., Wu, Q., Huang, B., Wang, G.Y., 2018. Numerical investigation of cavitation vortex dynamics in unsteady cavitating flow with shock wave propagation. Ocean Eng. 156, 424-434.

Wang, Y.W., Huang, C.G., Fang, X., Yu, X.N., Wu, X.C., Du, T.Z., 2016. Cloud cavitating flow over a submerged axisymmetric projectile and comparison between twodimensional RANS and three-dimensional large-eddy simulation methods. J. Fluids Eng. Transac. ASME 138 (6), 10.

Weiss, J.M., Smith, W.A., 1995. Preconditioning applied to variable and constant density flows. AIAA J. 33 (11), 2050-2057.

Weller, H.G., 2008. A New Approach to VOF-Based Interface Capturing Methods for Incompressible and Compressible Flow. OpenCFD Ltd., Rep. TR/HGW/04.

Yu, X.X., Huang, C.G., Du, T.Z., Liao, L.J., Wu, X.C., Zheng, Z., Wang, Y.W., 2014. Study of characteristics of cloud cavity around axisymmetric projectile by large eddy simulation. J. Fluids Eng. Transac. ASME 136 (5), 8. 